\title{
Shear modulus of two-dimensional colloidal crystals
}

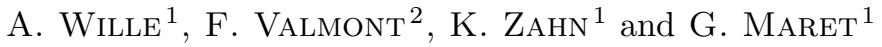 \\ 1 Universität Konstanz, Fachbereich Physik, Fach M 621 \\ D-78457 Konstanz, Germany \\ 2 Institut C. Sadron, CNRS - 6 rue Boussingault \\ F-67083 Strasbourg Cedex, France
}

PACS. 62.20.Dc - Elasticity, elastic constants.

PACS. 82.70.Dd - Colloids.

\begin{abstract}
A new method for the determination of elastic constants of colloidal systems is described. We study super-paramagnetic microspheres confined by gravity to a two-dimensional layer at a water-air interface. Under an external vertical magnetic field the particles arrange in a crystalline triangular phase because of the repulsive dipole-dipole interaction. By use of an optical tweezer, one triangle formed by three spheres is rotated from its equilibrium position and the relaxation time measured using video-microscopy. We demonstrate that this time is directly related to the shear modulus $\mu$ of the crystal and study $\mu$ as a function of the magnetic particle interaction strength.
\end{abstract}

During the last decades the interest in colloidal systems has grown tremendously because of their widespread technological applications and due to the availability of precisely calibrated particles used as model systems in "classical" condensed-matter physics [1]. The crystallization of colloids, both in two and three dimensions (2D and 3D), has been a continuous matter of particular interest. The research mostly focused on the analysis of structure and dynamics of colloidal systems on different length and time scales through static or dynamic light scattering techniques.

Elastic constants of colloidal crystals - essentially the shear modulus $\mu$ - were determined from the shear-induced resonance of the crystal through light scattering techniques (see [2] for recent work). As in real crystals, the value of $\mu$ is found to depend strongly upon the crystalline morphology and changes significantly between randomly oriented crystallites and shear-ordered samples [3]. In addition, using this method, only a very reduced number of modes can be investigated. A different approach to determine elastic constants is based on the analysis of the thermally induced vibrations of the particles in the crystal [4]. These fluctuations are inversely proportional to the elastic constants but depend also upon the system size studied. Therefore a finite-size scaling method is applied to extrapolate to the macroscopic elastic constants. We will show [5] that this method is well applicable to our system as described below and gives results in good agreement with theoretical predictions. However, so far the method can only be used for defect-free samples, a situation not always easy to realize in experiments. 

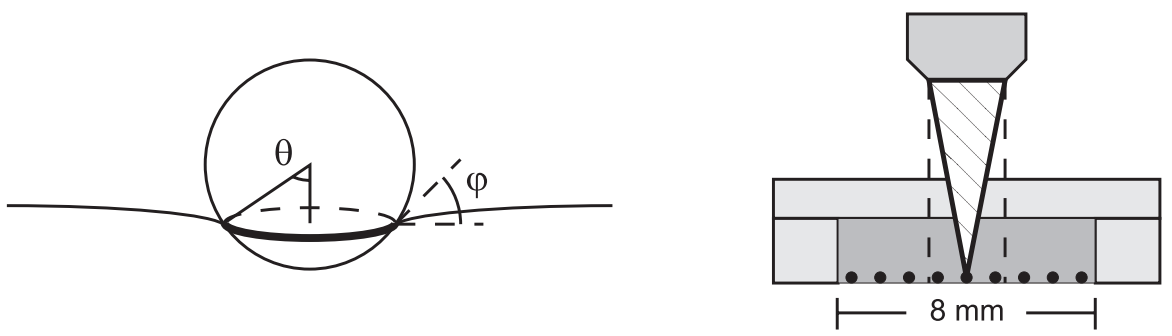

Fig. 1 - Left: deformation of the air-water interface due to the particle weight. For $\theta$ and $\varphi$ see text. Right: cross-section through the sample cell. The particles are confined due to the air-water interface and gravity.

In this work we describe a novel approach based on the use of optical tweezers to determine elastic constants in 2D. Since the work of Ashkin [6], the manipulation of individual or groups of colloidal particles trapped in a focused laser beam has become a widespread method in colloid- and bio-physics. We use optical tweezers to induce local deformations in a $2 \mathrm{D}$ colloidal crystal and show for the first time how elastic constants can be determined from the relaxation behavior of the deformation. The reliability of this method is demonstrated and our results for the shear modulus are in good agreement with theoretical predictions.

Experimental setup. - Aqueous solutions of monodisperse super-paramagnetic colloidal particles with a diameter of $4.5 \mu \mathrm{m}$ are used [7]. They consist of a porous polymer matrix with $\mathrm{Fe}_{2} \mathrm{O}_{3}$ grains embedded inside the pores. The iron oxide doping, of approximately $10 \%$ in volume, leads to a specific density of the particles of $1.5 \mathrm{~g} / \mathrm{cm}^{3}$. Therefore particles are subjected to strong sedimentation (velocity of the order of $6 \mu \mathrm{m} / \mathrm{s}$ ) and in our drop geometry described below arrange in a single layer at the water-air interface (compare fig. 1). A magnetic field $B$ is applied perpendicular to the interface inducing a magnetic dipole moment $M$ in the colloidal particles. The resulting repulsive interaction $E_{\text {magn }}$ is conveniently controlled by the magnetic field strength and is calibrated through the dimensionless interaction parameter $\Gamma$ :

$$
\Gamma=\frac{E_{\mathrm{magn}}}{k_{\mathrm{B}} T}=\frac{\mu_{0}}{4 \pi} \frac{(\chi B)^{2}(\pi \rho)^{\frac{3}{2}}}{k_{\mathrm{B}} T} .
$$

Here $\rho$ is the 2D particle density and $\chi$ is an effective magnetic susceptibility of the particles. The value of $\chi$ varied slightly among different particle batches and was of the order of $(7 \pm 0.7) \cdot 10^{-11} \mathrm{~A} \mathrm{~m}^{2} / \mathrm{T}$ [8]. A precise determination of $\chi$ is described in ref. [9]. In addition, within the experimental accuracy of $1 \%$ a perfect super-paramagnetic behavior, i.e. no magnetic hysteresis, is observed by comparing pair correlation functions (in the liquid) or Lindemann parameters (in the crystal) $[9,10]$.

The presented setup is quite special as the particles are confined at a water-air interface in a hanging drop geometry. In the following we will demonstrate that such a system is, in fact, an almost ideal 2D model system.

First, the deformation of the interface through the weight of the particles $F_{\mathrm{g}}=\mathrm{m} \cdot \mathrm{g}$ will be considered (see fig. 1). We define the angle $\varphi$ between the horizontal and the solvent interface at the circular line of contact between colloid and water. $\theta$ denotes the angle between the vertical axis and a vector pointing to the line of contact. The force caused by the surface tension $\gamma$ is $F_{\gamma}=2 \pi R \cdot \gamma \sin \theta$, obtained by integration of $\gamma$ over the line of contact. The vertical component of this force, which counterbalances the weight of the particle, is $F_{\gamma} \cdot \sin \varphi$. As the ratio of $F_{\mathrm{g}} / F_{\gamma}$ is only of the order of $10^{-6}$, the deformation perceptible by the angle 
$\varphi$ is in the range of $\mu \mathrm{rad}$. Even though the real physical situation is somewhat more complex as the particles are entirely wetted $(i . e . \theta=0)[11]$, the estimation of the order of magnitude remains valid and allows to neglect this effect completely.

Second, we consider the effect of capillary waves. Given the size of our cell, we can neglect inertial waves. The wave vector spectrum of the amplitude $\hat{\zeta}(\boldsymbol{q})$ is completely determined by the surface tension $\gamma$ and is given by

$$
\left\langle|\hat{\zeta}(\boldsymbol{q})|^{2}\right\rangle=\frac{k_{\mathrm{B}} T}{(2 \pi)^{2} \gamma q^{2}} .
$$

The mean-squared amplitude of the interface is now calculated as

$$
\left\langle\zeta^{2}\right\rangle=\int\left\langle|\hat{\zeta}(\boldsymbol{q})|^{2}\right\rangle \mathrm{d}^{2} q=\frac{k_{\mathrm{B}} T}{2 \pi} \int_{q_{\min }}^{q_{\max }} \frac{1}{\gamma q} \mathrm{~d} q=\frac{k_{\mathrm{B}} T}{2 \pi \gamma} \ln \left(\frac{q_{\max }}{q_{\min }}\right) ;
$$

$q_{\text {min }}$ is determined by the system size $\left(q_{\min }=2 \pi / 16 \mathrm{~mm}\right)$ and $q_{\max }$ is limited by the shortest possible wavelength, which we assume to be $1 \mathrm{~nm}$. We deduce that the mean amplitude $\left\langle\zeta^{2}\right\rangle^{1 / 2}$ is of the order of $1 \mathrm{~nm}$, i.e. entirely negligible on the length scale of the colloids.

Finally we consider the particle movement along the $z$-axis caused by the thermal energy $k_{\mathrm{B}} T$. Equating the latter with the potential energy difference of a particle in the gravitational field $k_{\mathrm{B}} T=m g \Delta z$ leads to a displacement of the order of $\Delta z=6 \mathrm{~nm}$ at room temperature. In fact, this is the most relevant effect (of the three discussed) and actually makes the simple method of confinement through gravity $(\Delta z \leq R)$ impossible for particle sizes below $1 \mu \mathrm{m}$. However, in our case $\Delta z$ is still small enough with respect to the diameter of the colloids making our setup basically an ideal $2 \mathrm{D}$ system.

Nevertheless, as illustrated by the last argument, a small height difference between the center and the border of the solvent drop (fig. 1) leads to a large difference in the potential energy compressing the system. For example, a height difference of only $1 \mu \mathrm{m}$ causes a density variation of $5 \%$ between the border and the center at a relatively high interaction strength of $\Gamma=220$. Therefore, for a proper performance of the experiments it was essential to develop a setup with an extremely flat interface. This was achieved by a special sample holder sketched in fig. 2. It consists of a quadratic cell made of quartz glass of $20 \mathrm{~mm}$ length and $2 \mathrm{~mm}$ thickness. In the center a cylindrical hole of $1 \mathrm{~mm}$ depth and $8 \mathrm{~mm}$ diameter is drilled into the cell. This void is filled with the colloidal suspension and serves as sample volume. Via a small drain it is connected to a second cylindrical hole of the same depth and diameter $4 \mathrm{~mm}$, which is used to change the amount of water in the sample volume. This is achieved by a syringe connected through a capillary tube to the small cylindrical hole. The inner side of the sample volume is hydrophilic while the lower flat surface of the cell is hydrophobic. Therefore, the drop of suspension wets the sample volume entirely and the lower edge of the larger cylindrical hole determines the boundary of the liquid drop. If, by means of the syringe, the amount of solvent in the volume is adjusted accordingly, a completely flat surface can be achieved. We obtain a flatness of less than $1 \mu \mathrm{m}$ over the entire interface. The syringe position and hence the interface curvature is automatically adjusted by a computer. As a control parameter we use the apparent size of the particles obtained by video-microscopic evaluation of the pictures taken by a CCD camera (fig. 2). In fact, the apparent size of the colloids changes when the particles move out of the focus of the microscope $(\times 100$ magnification, NA 0.8$)$.

The optical tweezer consists of a strongly focused laser beam which pulls dielectric particles into the maximum of their electromagnetic field, so that the particles remain trapped [6]. An argon-ion laser (Coherent INOVA 300) is focused through a set of two lenses onto the aperture 

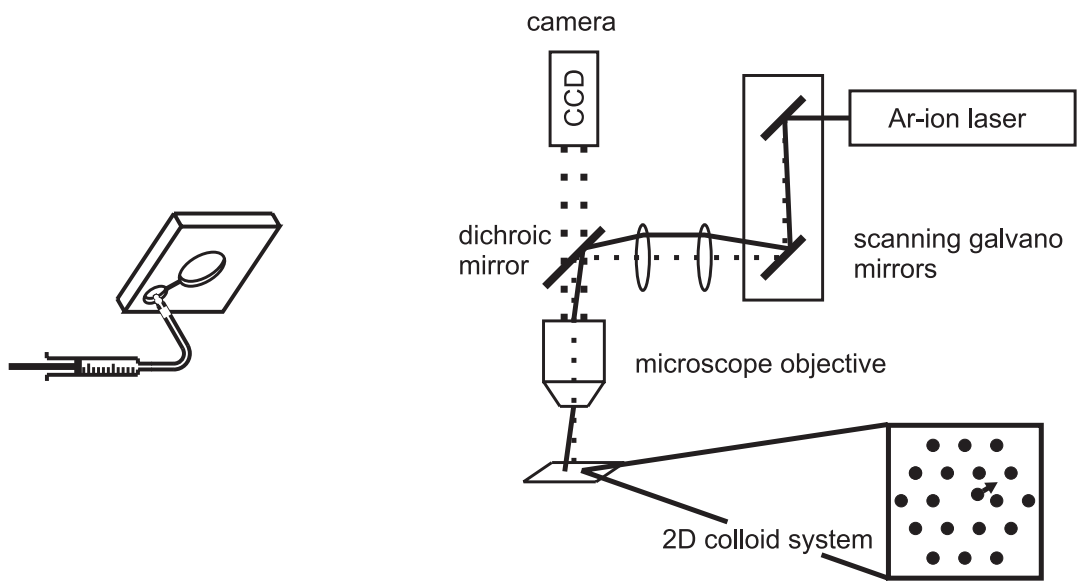

Fig. 2 - Left: bottom side of the cell. The large reservoir contains the sample. The small one is used to control the amount of water by a syringe. Right: the CCD camera takes images through the microscope objective which are analyzed by a computer to obtain the particle coordinates. The optical tweezer consists of an Ar ion laser which is focused into the objective by a dichroic mirror. The galvano mirrors enable the tweezer to move in the sample plane.

of the microscope objective. The latter is positioned in the focal plane of the second lens while a two-axis scanning galvano mirror is placed in the focal plane of the first lens. Thus, an angular variation of the laser beam obtained by a movement of the scanning mirror leads to a different incident angle of the beam on the objective aperture. In this way the laser focus can be moved to defined positions in the sample plane. In addition, as the mirrors can be modulated with a frequency of up to $500 \mathrm{~Hz}$ it is possible to manipulate several particles simultaneously.

Particular to our experiment is that the tweezer is only used for trapping within the horizontal plane, as the particles are already confined vertically by gravity to the water/air interface. The $2 \mathrm{D}$ trapping - as compared to $3 \mathrm{D}$ - substantially reduces the laser power necessary to manipulate the colloids. The typical power to tweeze one particle at $514 \mathrm{~nm}$ wavelength is $0.5 \mathrm{~mW}$. The application of a much higher laser power would in fact lead to thermal convection in the sample due to the absorption of the $\mathrm{Fe}_{2} \mathrm{O}_{3}$-doped particles.

Determination of the shear modulus. - The described setup was used to probe the shear modulus $\mu$ directly and - to our knowledge for the first time - on a microscopic length scale rather than by application of a macroscopic stress. Thus, our measurements give "ideal" values of $\mu$, i.e. for a defect-free solid, provided our derivation of macroscopic elastic constants from microscopic data is correct. Obviously for comparison with theory it is most useful to have ideal elastic constants rather than values determined by the (likely) complex topology of the lattice defects.

To probe the shear modulus in our 2D colloidal system we exploit the analogy of a rotated disk in an elastic continuum: three particles forming a triangle were rotated by the optical tweezer on a circle with constant radius (fig. 3). The relaxation of this displacement of the three particles into their equilibrium position was observed.

In detail, three adjacent particles lying on an equilateral triangle were displaced by 20 degrees on a circle around their center of mass by a stepwise tweezer rotation. The tweezer moved to a new destination (close enough to the actual particle position, such that the par- 

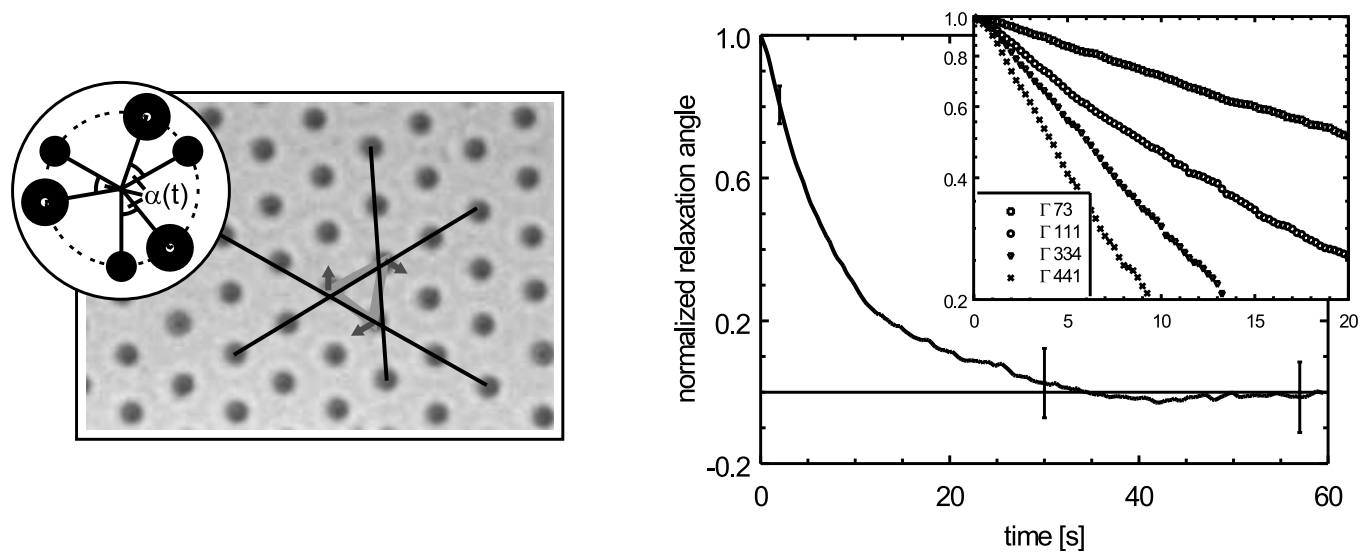

Fig. 3 - Left: the labeled particles were moved on a circle by the tweezer to induce a local deformation. Right: the time-dependent relaxation of the standardized deformation angle $\alpha$ for $\Gamma=279$ and four other crystal stiffnesses plotted logarithmically in the inset.

ticle is still sufficiently confined in the trap) and is kept there until the particle reaches the center position of the tweezer. This is done in turns for all three particles until the angular displacement is equal to 20 degrees. In fig. 3 such a twisted triangle is shown. Hereafter the tweezer is switched off and the particle relaxation is recorded in terms of the angle (line between a particle and the common center of the triangle compared to its equilibrium position) as a function of time. In the main part of fig. 3 a typical relaxation curve (normalized to 1 at $t=0 \mathrm{~s}) \mathrm{vs}$. time is shown obtained by averaging 277 measurements. This curve was obtained for $\Gamma=279$, i.e. deep in the crystalline phase (the melting transition occurs at $\Gamma=60[10]$ ). In the inset - in logarithmic scale - the dependence of the relaxation time $\tau$ upon the interaction strength $\Gamma$ is illustrated: as $\Gamma$ increases (which is equivalent to a decrease in the effective temperature) the crystal becomes stiffer resulting in a decrease of $\tau$. A complete set of curves was obtained for $\Gamma$ between 68 and 441 .

The following simple model enables us to determine the shear modulus from the relaxation of the angle $\alpha$ : We represent the rotated triangle as a twisted solid disk (of radius $R_{\mathrm{D}}=0.91 \cdot a$ [12]) in a $2 \mathrm{D}$ elastic continuum. In linear elasticity [13] the strain induced by a disk under a given torque $M$ is known to be inversely proportional to the shear modulus $\mu$ and decays with $1 / r^{2}$ from the center of the disk [14]: $\varepsilon_{r r}=\varepsilon_{\varphi \varphi}=0, \varepsilon_{r \varphi}=M /\left(\pi \mu r^{2}\right)$. The latter is related to the angular displacement $u_{\varphi}$ through the basic differential equation of $2 \mathrm{D}$ elasticity [14]

$$
2 \varepsilon_{r \varphi}=\frac{\partial u_{\varphi}}{\partial r}-\frac{u_{\varphi}}{r} \quad \text { solved by } \quad u_{\varphi}=-\frac{M}{\mu \pi r} .
$$

We set $u_{\varphi}=R_{\mathrm{D}} \cdot \alpha^{\prime}$ and integrate with respect to $\alpha^{\prime}$ from 0 to a finite value $\alpha$. Using $\int_{0}^{\alpha} M \mathrm{~d} \alpha^{\prime}=E$ (the energy of the deformation), we derive

$$
E=2 \pi \mu \alpha^{2} R_{\mathrm{D}}^{2}
$$

This implies a repel force $F_{\mathrm{R}}$ proportional to the twist angle $\alpha$ :

$$
F_{\mathrm{R}}=-4 \pi \mu R_{\mathrm{D}} \alpha .
$$

If we assume that the colloidal movement is totally overdamped due to the high viscosity of the suspension and if we neglect hydrodynamic interactions, we can equate $F_{\mathrm{R}}$ with the 


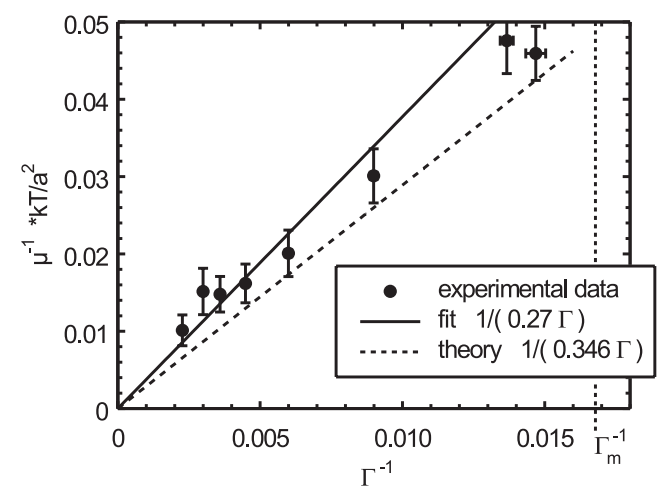

Fig. $4-$ The inverse shear modulus $\mu$ is plotted vs. $\Gamma$. The dots indicate the measured values with error bars. The solid line is the fit of the experimental data and the dashed line represents the theoretical behaviour.

Stokes friction:

$$
F_{\mathrm{S}}=-6 \pi \eta R_{\mathrm{P}} \frac{a}{\sqrt{3}} \frac{\partial \alpha}{\partial t} .
$$

There $\eta$ denotes the viscosity of water [15] and $R_{\mathrm{P}}=2.25 \mu \mathrm{m}$ is the particle radius. The resulting differential equation $\left(F_{\mathrm{R}}+3 \cdot F_{\mathrm{S}}=0\right)$ has the solution:

$$
\alpha(t)=\alpha_{0} \cdot e^{-t / \tau}, \quad \tau=\frac{\eta R_{\mathrm{P}}}{\mu} \sqrt{\frac{9 \pi}{2 \sqrt{3}}} .
$$

The obtained experimental relaxation curves (like fig. 3) are fitted to $\alpha(t)=\exp [-t / \tau]$ and $\mu$ is deduced, $\mu$ being the only unknown in eq. (8). Figure 4 shows the dependence of the shear modulus on $\Gamma$.

Discussion. - As the interaction potential is known exactly, the elastic constants can be obtained by standard formulas of elasticity theory, at least for zero temperature [16]. For the shear modulus one obtains $\mu=0.346 \Gamma$. If we fit a straight line to our data (solid line in fig. 4) a slope of 0.27 is found for high $\Gamma$, respectively, low temperatures. Considering that this value is obtained without any adjustable parameter, this is a rather excellent agreement between theory and experiment. Nevertheless, some systematic deviation from the straight line is observed for small $\Gamma$ because close to the phase transition temperature $\Gamma_{m}^{-1}$ the zerotemperature model is not valid. We will discuss the behavior of the shear modulus and the bulk modulus in the vicinity of the melting transition in a separate paper.

Finally some points remain to be clarified: On the one hand the radius of the disc $\left(R_{\mathrm{D}}\right)$ is not precisely defined and could be varied by a factor of order 1 . On the other hand, it is not clear whether the zero-temperature calculation may be extended to the temperature range where the experiments have been performed. Finally the measurement is performed on a local scale and even though we probe the shear modulus, the precise relationship between our results and the macroscopic elastic constant $\mu$ is a priori not clear. This issue will be addressed in a forthcoming paper [5] on 2D colloidal crystals using a finite-size scaling scheme, recently proposed and applied to 2D ensembles of hard discs by Sengupta et al. [4].

Conclusion. - In summary, we have demonstrated in detail that our system of paramagnetic colloids disposed at a water-air interface is an almost ideal 2D model system. We showed 
that optical tweezers can be used to manipulate several particles simultaneously and move them against the repel forces of the surrounding neighbours to defined positions in the lattice. We applied a defined strain to the lattice and were able to study the relaxation to equilibrium. A simple model was developed to extract the shear modulus $\mu$ from the relaxation time and good agreement with a zero-temperature calculation was obtained. Our method can be used in other relaxation modes to determine a complete set of elastic constants (shear modulus $\mu$ and bulk modulus $B$ ). This enables a crucial quantitative testing of the $2 \mathrm{D}$ melting theory by Kosterlitz and Thouless (KTHNY), which relates the melting temperature to $\mu$ and $B$. In addition our method can be used to probe the orientational stiffness (equivalent of the Frank constant of the nematic liquid crystals) in the hexatic phase which plays an important role in the KTHNY theory.

$$
* * *
$$

Financial support by the Deutsche Forschungsgemeinschaft (DFG) in the frame of the Sonderforschungsbereich 513 and of the Deutscher Akademischer Austauschdienst (DAAD) is acknowledged.

\section{REFERENCES}

[1] Pusey P., Liquids, Freezing and the Glass Transition (North-Holland, Amsterdam) 1991.

[2] Schöpe H., Decker T. and Palberg T., J. Chem. Phys., 109 (1998) 10068.

[3] Palberg T. et al., J. Phys. III, 4 (1994) 457.

[4] Sengupta S., Nielaba P., Rao M. and Binder K., Phys. Rev. E, 61 (2000) 1072.

[5] ZAHN K. et al., in preparation (2001).

[6] Ashkin A., Dziedzic J., Bjorkholm J. and Chu S., Opt. Lett., 11 (1986) 288.

[7] Dynabeads M-450, uncoated; Deutsche Dynal GmbH Postfach 111965 D-20419 Hamburg.

[8] Within a given batch, however, the value of $\chi$ varies by less than $5 \%$.

[9] Zahn K., Méndez-Alcaraz J. and Maret G., Phys. Rev. Lett., 79 (1997) 175.

[10] Zahn K., Lenke R. and Maret G., Phys. Rev. Lett., 82 (1999) 2721.

[11] This can be shown by turning the sample holder upside down (now air on the top, water on the bottom), when the particles are found to sediment away from the water-air interface. If the colloids were only partially wetted, the resulting force due to the surface tension would maintain them fixed to the interface.

[12] This is an effective radius which corresponds to the circle area that equals the area of the Voronoi cells of the three tweezed particles: $R_{\mathrm{D}}=a \cdot \sqrt{3 \cdot \sqrt{3} / 2 \pi}$.

[13] Our use of linear elasticity is rationalized as follows: We simulated a hexagonal 2D lattice of dipoles at zero temperature and calculated the potential energy of the deformation sketched in fig. 3 depending on the angle $\alpha$. In a polynomial fit of $E(\alpha)$ taking orders higher than the quadratic one in $\alpha$ into account, the coefficient of $\alpha^{2}$ shifts by $4 \%$. This would lead to corrections in the measured shear modulus which are much smaller than the errors of the experimental data. Therefore we neglect terms of fourth and higher order in the deformation energy.

[14] Hahn H., Elastizitätstheorie (B. G. Teubner, Stuttgart) 1985.

[15] Due to the interface the Stokes diffusion of the particles is increased by $8 \%$ compared to bulk [9]. This is considered by a decrease of the effective viscosity.

[16] Kusner R., Ph.D. thesis, Case Western Reserve University (1993). 